\title{
Time to Diagnosis and Factors Affecting Diagnostic Delay in Amyotrophic Lateral Sclerosis
}

\author{
Danielle Richards $^{1} \bullet$ John A. Morren ${ }^{1} \bullet$ Erik P. Pioro $^{1,2}$ \\ ${ }^{1}$ Neuromuscular Center, Neurological Institute, Cleveland Clinic, Cleveland, OH, USA; \\ ${ }^{2}$ Department of Neurosciences, Lerner Research Institute, Cleveland Clinic, Cleveland, \\ $\mathrm{OH}, \mathrm{USA}$
}

Author for correspondence: John A. Morren, Neuromuscular Center, Neurological Institute, Cleveland Clinic, Cleveland,OH, USA. E-mail: morrenj@ccf.org Doi: https://doi.org/10.36255/exonpublications.amyotrophiclateralsclerosis. diagnosticdelay. 2021

\begin{abstract}
At present, disease-modifying treatments for Amyotrophic Lateral Sclerosis (ALS) remain limited, with early intervention crucial for maximum potential benefit. A majority of patients will develop dysphagia during the course of their disease, and most will die within three years of the first symptom onset due to respiratory complications. Therefore, early diagnosis is vital to ensure the patient receives appropriate multidisciplinary care and resultant improved longevity as well as quality of life. However, a recent literature review found that ALS patients experience a diagnostic delay of 10-16 months from symptom onset. This chapter examines the factors that contribute to diagnostic delay and potential interventions to decrease time to diagnosis.
\end{abstract}

Keywords: amyotrophic lateral sclerosis; diagnostic delay; Lou Gehrig's disease; misdiagnosis; motor neuron disease; time to diagnosis

In: Amyotrophic Lateral Sclerosis. Araki T (Editor), Exon Publications, Brisbane, Australia. ISBN: 978-0-6450017-7-8. Doi: https://doi.org/10.36255/exonpublications. amyotrophiclateralsclerosis. 2021

Copyright: The Authors.

License: This open access article is licenced under Creative Commons Attribution-NonCommercial 4.0 International (CC BY-NC 4.0) https://creativecommons.org/licenses/by-nc/4.0/ 


\section{INTRODUCTION}

Currently, for patients diagnosed with amyotrophic lateral sclerosis (ALS), disease modifying treatments remain limited. The typical disease course is marked by a subtle onset and insidious progression, with patients experiencing variable degrees of weakness, spasticity, and muscle atrophy, ultimately resulting in progressive deterioration of limb use, ambulation, speech, swallowing, and breathing. A majority of ALS patients will develop dysphagia during the course of the disease as a result of disease progression involving the bulbar musculature and most will die within three years of the first symptom onset due to respiratory complications (1). This makes early diagnosis and subsequent referral to an appropriate tertiary neuromuscular center/ALS clinic crucial to assure the patient receives appropriate multidisciplinary care and the resultant improved longevity and quality of life. It therefore becomes critical to understand where along the disease timeline diagnostic delays occur and what factors contribute to its prolongation.

\section{DIAGNOSTIC DELAY IN NON-NEUROLOGICAL AMBULATORY MEDICINE}

Broadly speaking, diagnostic delay impacts medicine across the spectrum of diseases and subspecialties. In a 2014 study of celiac patients, diagnostic delay of greater than 10 years was reported in $32 \%$ of patients surveyed (2). In a 2012 study of missed or delayed diagnoses of breast and colon cancer, which have the potential for positive outcomes if caught early, Poon et al. found that 95\% of diagnostic delay involved physician 'cognitive error', which was defined as errors arising from inadequate clinical knowledge or poor clinical judgment. Forty-six percent of these cognitive errors involved an inappropriate workup strategy and $53 \%$ were related to misinterpretation of results. In $66 \%$ of cases reviewed, researchers found that appropriate application of management guidelines for breast and colon cancer could have prevented further delay (3).

Another meta-analysis of misdiagnosis in various cancer subtypes found a majority of breast cancer diagnostic delay was related to similar cognitive errors of mammogram radiology reviews, where general radiologists lacked specialized training to appropriately assess mammogram studies. Melanoma diagnoses were similarly delayed or altogether missed secondary to a lack of physician experience with or clinical knowledge of the disease (4).

In a 2006 study regarding medical malpractice cases, the most common causes of missed or delayed diagnosis, in descending order, was a failure to order appropriate diagnostic testing, inadequate follow-up plans, failure to collect an accurate history and physical exam, and finally incorrect interpretation of diagnostic testing (5), suggesting limited medical knowledge to be a significant factor in delayed diagnosis. Such delays ultimately result in inappropriate utilizations of resources, patient harm, and potential damage to the physician-patient relationship. 
Overall, across a broad spectrum of medical subspecialties, there arise clear similarities in factors that result in diagnostic delay, and the field of ALS is unfortunately no exception. However, there may be more in common when the comparison is between ALS and degenerative conditions with similarly guarded prognosis.

\section{DIAGNOSTIC DELAY IN CHRONIC NEURODEGENERATIVE DISEASE}

Similar factors of diagnostic delay are readily found in essentially all subspecialties of neurology, but here we will limit our discussion to dementia, which encompasses a wide variety of chronic neurodegenerative diseases and remains a field where early diagnosis and subsequent medical and social intervention remains paramount for appropriate management. In a 2012 meta-analysis by Aminzadeh et al., only about $50 \%$ of cases of mild to moderate dementia were ever correctly diagnosed, with first notable diagnostic delay occurring between symptom onset and initial physician consultation; family members would frequently wait one to two years before seeking any medical assessment. Furthermore, additional delays were caused by subsequent referrals to specialists, as the initial consulted physician was unlikely to be the provider to make the ultimate diagnosis (6).

Research has also noted that a significant barrier to early diagnosis is the limited clinical encounter time often seen with primary care visits, hampering the ability to perform detailed-enough exams, determine appropriate tests, or procedures to detect dementia (6-8). Moreover, even when dementia was suspected, primary care physicians/providers (PCPs) have expressed hesitation about providing the correct diagnosis; they assumed patients and/or their relatives would not want to know, and they even questioned what effect the diagnosis would have on the PCP-patient dynamic (7).

In addition to limited time available for appropriate assessment and workup, Aminzadeh et al. reported other causes for diagnostic delay including limited medical knowledge regarding the disease course, deficits in communication and management skills, and a problematic attitude they termed "therapeutic nihilism" (6). This mindset encapsulates an overall negative view or stigma held by physicians towards dementia and has appeared elsewhere in the literature. In prior studies, physicians have expressed concerns that a diagnosis of dementia would do more harm than good. There was a general perception that there are no available or effective treatments to slow the progression of disease and therefore such a work up would not be worthwhile $(7,8)$. Similarly, patients' relatives/caregivers reported one of the most common causes of delayed diagnosis related to physician attitudes. In one study, 33\% of relatives/caregivers reported that the initial assessing physician did not consider anything to be abnormal with the patient and in another $7 \%$ of cases, physicians told relatives/caregivers that pursuing a diagnosis would not be worthwhile (9).

Overall, one can already begin to anticipate similar factors that affect time to dementia diagnosis such as limited physician knowledge or disease stigma being similarly applicable to ALS. 


\section{LENGTH OF DIAGNOSTIC DELAY IN ALS}

A recently published article that reviewed twenty-one retrospective studies of time from symptom onset to correct diagnosis in the ALS patient population between 1990 and 2020 found that ALS patients experience a delay of about 10-16 months from symptom onset to diagnosis (Figure 1) (10).

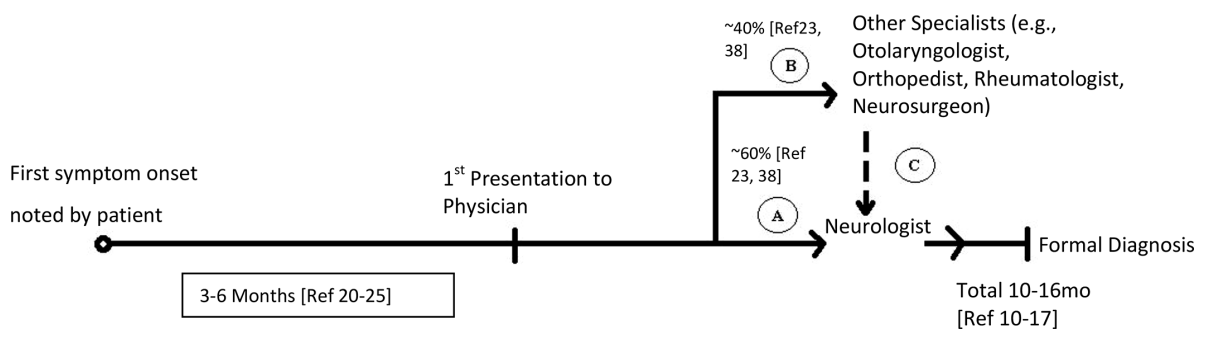

Figure 1. Pathway to ALS diagnosis from first symptom onset to final diagnosis as reported in Richards et al. The initial delay to be evaluatied by the first provider averages 3-6 months. About $60 \%$ of patients are then referred to neurologists while the remaining $40 \%$ are referred to non-neurologists. Of note, in some studies presenting to a neurologist compared to a non-neurologist does not seem to increase diagnostic delay when the neurologist is the first consultant [Ref 23] or even the third consultant (Ref 21).

This has been confirmed in other research as well (11-14), including more recent studies in 2020 that found a median delay of about 12 months (15-17) and a mean delay of 17 months (15). In the Richards et al. article, the longest delay was 27 months, reported in a study reviewing the United States Centers for Medicare \& Medicaid Services database (18) and shortest reported median interval was from a study of a national database used by tertiary ALS clinics in France, reporting a delay of 9.1 months (19).

\section{PATIENT-SPECIFIC FACTORS LEADING TO DIAGNOSTIC DELAY}

Much like dementia, the first delay occurs between symptom onset and patients seeking medical attention. Generally, ALS patients will wait anywhere from three (20) to almost six months (21-25) after symptom onset before undergoing a medical evaluation, but other patient-specific factors contribute to the diagnostic timeline (Table 1).

\section{Disease phenotype and diagnostic delay}

Clinical presentation plays a significant role in time to diagnosis, particularly with regards to bulbar versus spinal-onset presentation $(8,13,15,16,18,19,21,22$, 25-31), with the literature suggesting patients with bulbar-onset ALS experience a delay to diagnosis three to seven months shorter than those with spinal-onset ALS $(21,26-28)$. The study reporting the longest delay of 2.25 years, as 


\section{TABLE 1 Patient and Physician/Provider factors found to affect time to diagnosis in ALS (10)}

\begin{tabular}{ll} 
Patient Factors & Physician/Provider Factors \\
\hline Age & "Cognitive Errors"/Misdiagnosis \\
Gender & Inappropriate testing/lack of testing \\
$\begin{array}{l}\text { Comorbidities } \\
\begin{array}{l}\text { Phenotype (region of onset, presence } \\
\text { of visible fasciculations) }\end{array}\end{array}$ & Initial referral to neurologist vs non-neurologist \\
\end{tabular}

mentioned in the review paper by Richards et al. (10), showed a substantial difference between delays in the bulbar-onset group ( 1.25 years) compared to the spine-onset group (2.5 years) (18). Of note, for patients presenting with both bulbar and spine symptoms, the median time to diagnosis dropped to 0.25 years $(10,18)$. Patients with spinal-onset presentation also receive more differential diagnoses than those with bulbar presentation (26) and are more likely to be misdiagnosed (27). Compared to spine-onset patients, bulbar-onset patients are also more likely be assessed by a neurologist and less likely by a PCP or orthopedist (28). Multiple studies have also noted shorter diagnostic delay to be associated with shorter survival time $(11,12,17,32-36)$, likely in part driven by the patients with a more rapidly progressive disease who may seek medical attention sooner than those with a more insidious course $(12,32)$ and bulbar patients who generally experience a more rapid course than their spinal-onset counterparts.

One study by Scialo et al. divided their patient population into two subtypes: those with a diagnostic delay of less than or greater than 36 months. They found that the cohort with a diagnostic delay of greater than 36 months were more likely to present with an atypical clinical phenotype. In another study, patients who presented with clinically-evident fasciculations also experience a shorter delay to diagnosis, though not as short as the bulbar-onset population (23).

\section{Age of onset and diagnostic delay}

Multiple studies have also noted age as a factor that prolongs diagnostic delay $(22,24,26,37)$. In one study, patients 65 to 74 years old experienced longer times to diagnosis compared to those 55 to 64 years old, at 12 months and 8 months, respectively (26). Similarly, another study found that the median time to diagnosis was 12.4 months for patients over the age of 60 years compared to 8 months for younger patients. Furthermore, this study showed that diagnostic delay greater than 12 months was about 11 times more likely for patients in the $>60$ years age group (38). Patients over the age of 60 were also more likely to be initially misdiagnosed compared to younger populations (24). This age-related delay was not consistently found, with Nzwalo et al. reporting significant delay among the younger patient population, which they defined as less than 45 years of age (37). In one Italian multicenter study, older patients were noted to have a shorter diagnostic delay, which authors argued was likely due to more rapid 
disease progression as they are also more likely to present with a bulbar phenotype than younger populations (34), as similarly noted by Yates et al. (12). Interestingly, a study by Martinez-Molina et al. found no association with age and length of delay (16).

\section{Gender and diagnostic delay}

There is also some evidence of gender differences in time to diagnosis, with male patients experiencing longer delays than females $(37,38)$ though this could be related to the female predominance of bulbar-onset ALS (38). In one study, men were also more likely to receive a misdiagnosis compared to women by a ratio of 2.5 to 1 (25), though again this may be because bulbar-onset ALS has a higher female predominance. Interestingly, a study by Iwasaki et al. noted that diagnostic delay in male bulbar-onset patients was 10.5 months compared to 9.8 months in female bulbar-onset patients. In contrast, male spine-onset patients experienced a delay of 13.7 months versus 14.8 months for female spine-onset ALS patients. Martinez-Molina et al. again found no significant association with gender and length of delay (16).

\section{Patient comorbidities and diagnostic delay}

Another factor affecting time to diagnosis is the presence of other neurological comorbidities, particularly those diseases with symptoms similar to those of ALS $(16,18,23)$, or an overall complex medical history (39). In one study, the presence of comorbidities was associated with nearly twice the length of delay compared to patients without comorbidities, at 19.7 months and 11.1 months respectively (38). Delays have also been reported with patients presenting with frontotemporal dementia (FTD) as the predominant feature of their ALS-FTD, such as two patients in the Househam and Swash study who initially presented with dementia and ultimately experienced a 31.5 month delay from time of first physician assessment to diagnosis of ALS (25).

\section{PHYSICIAN/PROVIDER-SPECIFIC FACTORS LEADING TO DIAGNOSTIC DELAY}

As stated before, the first delay in the diagnostic timeline is the period between from disease onset to the patient seeking medical attention, a step that could be argued is generally independent of physicians and providers. However, once the patient is first assessed by a healthcare professional, there arise further physician dependent factors that prolong the time to diagnosis (Table 1).

\section{"Cognitive errors", misdiagnosis, and diagnostic delay}

Indeed, it becomes evident in the discussion above regarding patient specific factors that a great deal of this diagnostic delay is likely compounded by, if not a result of, physician 'cognitive error', which was above defined in 
non-neurological ambulatory medicine literature as errors stemming from providers' inadequate clinical knowledge or poor clinical judgment, such as inappropriate workup strategy or misinterpretation of results (3). There seems to be an apparent lack of clinical knowledge among physicians regarding spinalonset ALS, leading to diagnostic delay. This compares to the bulbar-onset patients, or patients presenting with fasciculations, which perhaps raise red flags more broadly known among physicians as ALS symptoms. Two thirds of PCPs self-report that their degree of training regarding ALS is low, with many expressing an overall lack of knowledge of disease clinical signs and symptoms (40). While neurologists are certainly more likely to be exposed to ALS during the course of their clinical experience, they too are at risk of making cognitive errors. In one study, Li et al. asked neurologists in multiple countries to rank the diagnostic importance of MND clinical features and then diagnose known MND case summaries. While in agreement on major MND characteristics, neurologists differed significantly with regard to their final diagnoses of the case summaries. Seemingly, the neurologists may have agreed in "theoretical terms", but applied this diagnostic knowledge in fundamentally divergent ways based on personal clinical experience (41). Misdiagnosis was the another factor that resulted in further delay, with incorrect diagnoses occurring in $13-68.4 \%$ of cases $(13,20-22,24,25,37,42)$. Such incorrect diagnoses included cerebrovascular disease, cervical myelopathy, neuropathy, radiculopathy, vertebral disc herniation, and myasthenia gravis, among many others (Table 2) $(20-22,25,38,42)$. A pertinent question that then arises is just who is making these misdiagnoses? Surprisingly, anywhere from $7-44.4 \%$ of misdiagnoses were made by neurologists $(13,20,25,42)$ with one study finding motor neuron disease (MND) was listed as an initial differential diagnosis in only $30.6 \%$ of ALS patients' medical records (38). According to one study, if neurologists are the first providers to assess the patient, only $56 \%$ correctly diagnosed ALS; interestingly, this increased to $78 \%$ if they were the second provider. However, it should be noted that this rather starkly contrasts with the $1 \%$ of patients correctly diagnosed by a primary care provider or other specialist during initial presentation (37). Misdiagnoses leads to more significant delay than those without any diagnosis at all $(13,20,21,24,42)$, with patients often only receiving the correct the diagnosis once their disease has further progressed (42).

\section{Specialist referrals and diagnostic delay}

While the first provider assessment is most frequently with the patient's PCP (13, $15,21,23,37)$, subsequent referrals to other specialists results in additional delays, with some research showing that neurologists make up $60 \%$ of initial specialist referrals $(23,38)$. Nzwalo et al. reported that $56 \%$ of cases will undergo subsequent neurology referral at some point during the course of the disease (37). This was supported by another study that found that while neurologists made up only $28 \%$ of the first specialist referral, $62 \%$ of patients received a neurology consult at some point within the first three referrals (21). In another study, $49 \%$ of ALS patients were referred to other specialists prior to a neurologist, with $54 \%$ of this group having been seen by otorhinolaryngologists (30). Other non-neurology 


\section{TABLE 2 Rates of specific misdiagnoses prior to formal diagnosis of ALS (10)}

\begin{tabular}{|c|c|c|}
\hline Study (Reference) & $\begin{array}{l}\text { Overall misdiagnosis } \\
\text { rate }(\%)\end{array}$ & Specific misdiagnosis subcategory rate (\%) \\
\hline Palese et al. (38) & $49 / 134(36.6 \%)$ & $\begin{array}{l}\text { Myelopathy (14.3\%), Radiculopathy (8.2\%), Stroke/ } \\
\text { Vascular encephalopathy (8.2\%), Neuropathy } \\
\text { unspecified (8.2\%), Nothing pathologic (6.1\%), } \\
\text { Arthrosis (6.1\%), Myasthenia gravis (6.1\%), } \\
\text { Carpal tunnel syndrome (4.1\%), Herniated } \\
\text { disc (4.1\%), Upper airway infection (4.1\%), } \\
\text { Musculoskeletal (4.1\%), Other (26.5\%) }\end{array}$ \\
\hline Galvin et al. (21) & $20 / 155(13 \%)$ & $\begin{array}{l}\text { Structural (65\%): Cerebrovascular disease, Hiatus } \\
\text { hernia with reflux, Cervical myeloradiculopathy, } \\
\text { and Lumbar radiculopathy. }\end{array}$ \\
\hline Paganoni et al. (22) & $158 / 304(52 \%)$ & $\begin{array}{l}\text { Neuropathy (28\%), Spine Disease (18\%), Vascular } \\
\text { (11\%), Neurodegenerative Disease (11\%), NMJ } \\
\text { disorder ( } 9 \%) \text {, ENT disorder (7\%), Muscle } \\
\text { Disease }(6 \%) \text {, other }(10 \%) .\end{array}$ \\
\hline $\begin{array}{l}\text { Belsh and } \\
\quad \text { Schiffman (42) }\end{array}$ & $14 / 33(42.4 \%)$ & $\begin{array}{l}\text { Radiculopathy (12.1\%), brachial plexus neuropathy } \\
\text { (9\%), Multiple Sclerosis (3\%), Myelopathy (3\%), } \\
\text { Polyneuropathy (3\%), Stroke (3\%), Depression } \\
\text { (6\%), Occult carcinoma (6\%), Pulmonary } \\
\text { emphysema (6\%), Congestive heart failure (3\%), } \\
\text { Drug induced dysarthria (3\%). }\end{array}$ \\
\hline Chiò (23) & $90 / 201(45 \%)$ & $\begin{array}{l}\text { Discal herniation/medullar compression (12\%), } \\
\text { Arthrosis/periarthritis ( } 9 \%) \text {, Narrow medullar } \\
\text { canal (4\%), Cerebrovascular accident (3\%), } \\
\text { Osteoporosis (2\%), Laryngitis/chronic tonsillitis } \\
\text { (2\%), Thyroid dysfunction (1\%), Parkinson's } \\
\text { disease (1\%), Multiple sclerosis (1\%), } \\
\text { Other (10\%). }\end{array}$ \\
\hline Cellura et al. (20) & $81 / 260(31.1 \%)$ & $\begin{array}{l}\text { Herniated disc/Cervical myelopathy (32.0\%), } \\
\text { Vascular pseudobulbar palsy (20.0\%), } \\
\text { Neuropathy/ Myopathy (8.6\%), Myasthenia } \\
\text { gravis (7.4\%), Carpal tunnel syndrome (6.2\%), } \\
\text { Depression (6.2\%), Alzheimer's dementia (5\%), } \\
\text { Parkinson disease (5.0\%), Arthrosis (2.4\%), } \\
\text { Thyroid dysfunction (2.4\%), Multiple sclerosis } \\
\text { (2.4\%), Stroke (1.2\%), Essential tremor (1.2\%). }\end{array}$ \\
\hline $\begin{array}{l}\text { Househam and } \\
\text { Swash (25) }\end{array}$ & $39 / 57(68.4 \%)$ & $\begin{array}{l}\text { Vocal cord dystonia, Depression, Laryngeal } \\
\text { cancer, Stroke (8.6\%), Stress, Thyroid disease, } \\
\text { Muscular dystrophy, Frozen shoulder (5.7\%), } \\
\text { asthma (5.7\%), Cervical Spondylosis, Arthritis } \\
\text { (14.3\%), Cramps, Heart disease, Trapped nerve } \\
\text { (8.6\%), Recurrent throat infection, Ear infection, } \\
\text { Medication side effect, Ligamentous strain, } \\
\text { Cervical disc prolapse, Peripheral neuropathy. }\end{array}$ \\
\hline
\end{tabular}


specialist referrals included orthopedists, rheumatologists, and neurosurgeons $(23,38)$, as well as physiotherapy and psychiatry $(25)$.

There are also further differences in length of delay secondary to specialist referral dependent on the type of subspecialist. Palese et al. reported a longer diagnosis delay for patients assessed by a non-neurologists (13 months) compared to those seen by a neurologist (10 months) (38). Nzwalo et al. also reported significantly reduced diagnostic delays for patients who underwent a neurology referral (37). In a study by Househam and Swash, ALS patients who were first referred to a neurologist experienced a shorter delay (10.2 months) compared to those referred to another subspecialty (12.3 months) (25). This was echoed in a later study that found prolonged times to diagnosis among patients referred to non-neurologists (39). Interestingly, spinal-onset patients referred to an orthopedist experienced an additional delay of 10 months compared to those referred to a neurologist (28). Those with bulbar onset experienced a delay to diagnosis of 4.9 months if the referral was to a neurologist, compared to 12.2 months for other specialists (collectively). Diagnostic delay was even more prolonged for patients referred to ENT in particular, at 24.7 months (13).

Differences in delay among subspecialties was not a unanimous finding. Turner et al. reported that a subspecialty referral, specifically to otorhinolaryngologists, did not subsequently result in significant diagnostic delay (30). One study found lower costs associated with neurology referrals but not a significant difference in time to diagnosis (21). In another study, there was no significant difference in diagnostic delay if a neurologist was the first or second physician seen, but this increased when they were seen as the third, fourth, or fifth provider, with time to diagnosis of 17, 19, and 21 months respectively (23). Matharan et al. noted a diagnostic delay roughly ten months longer for those seen by a neurologist versus non-neurologist. Interestingly, they also noted that there was no significant difference in delay depending on whether the patient was referred to a neurologist or sent home without further workup. Authors theorized, at least for the former, this could be due to patients early in the disease course needing serial EMGs or clinical examinations before the disease was advanced enough to be more definitively diagnosed. They also note that bulbar-onset ALS, which showed a shorter diagnostic delay, was more likely to be referred to ENT rather than a neurologist, potentially skewing the specialist referral data (15).

\section{Inappropriate/incomplete testing and diagnostic delay}

As stated earlier, those with spinal-onset presented received more differential diagnoses than those with bulbar-onset presentation (26) and are more likely to be misdiagnosed (27). Therefore, it is not surprising that those in the spine-onset subgroup were also more likely to undergo further diagnostic testing, including electrodiagnostic testing (EDX) comprising nerve conduction study (NCS) and electromyography (EMG), as well as neuroimaging such as MRI and CT scans (18). While EDX is certainly an appropriate step in working up MND (as will be further discussed below), neuroimaging which also has a role in ALS investigations may result in incidental findings that potentially introduce confounders and may add unnecessary procedures, thereby prolonging the diagnostic timeline. 


\section{Role of electrodiagnosis and neuroimaging in diagnostic delay}

While original El Escorial criteria did not allow for EMG findings to serve as a surrogate for clinical features of LMN degeneration, subsequent diagnostic criteria revisions have improved upon this. The 2006 Awaji-Shima criteria now permits fasciculation potentials (without need for positive sharp waves or fibrillation potentials) in the presence of chronic motor axon loss changes as adequate evidence of lower motor neuron degeneration, allowing for earlier diagnosis and classification. Furthermore, these Awaji-Shima criteria exhibit increased sensitivity (43) and equal specificity of an ALS diagnosis when compared to the revised El Escorial criteria (44). In a study by Palese et al., EDX was the most common first investigatory procedure in the pathway to the ALS diagnosis, followed by brain and spinal cord imaging (38). Research suggests that ultimately $75-100 \%$ of $\operatorname{ALS}(15,21,30)$ patients will undergo neurophysiologic/EDX testing at some point during their diagnostic path and $61-100 \%$ of patients will undergo brain MRI $(21,30)$.

Of course, the use of EDX testing does not guarantee a correct diagnosis. Evidence suggests EDX diagnostic sensitivities are lowest in patients categorized as possible ALS and intermediate in patients with probable and probable with laboratory support ALS; the highest sensitivities were found in those with definite ALS (43). In addition, other neuromuscular diseases such as multifocal motor neuropathy or Lambert-Eaton myasthenic syndrome may require a neurologist with a higher degree of expertise in EDX to collect and interpret the data in order to distinguish diseases such as these from ALS. Physicians should always be mindful of any alternative diagnoses to explain patients' presenting symptoms and exam features. Furthermore, results are not absolute and the lack of definite evidence for MND during one investigatory work up does not necessarily predict that future investigations will be similar.

\section{Surgical intervention and diagnostic delay}

Misdiagnosis as well as unnecessary or incomplete workups can unfortunately result in exposure to unnecessary procedures. Patients who are misdiagnosed more likely to undergo surgeries as a result with about $12-13 \%$ of ALS patients undergoing an inappropriate surgical procedure prior to their correct diagnosis $(27,45)$. This not only results in increased potential risk but also further delay $(27,37,38)$. In one study for example, the $12 \%$ of patients who underwent surgery prior to receiving an ALS diagnosis experienced an additional delay of roughly six months compared to the $43 \%$ who underwent medical management (27), thereby further compounding upon the misdiagnosis delay mentioned above. Of note, in one study of ALS patients who underwent inappropriate surgery prior to diagnosis, 32\% had a pre-operative EMG and of these patients, $72 \%$ of reports documented evidence of polyradiculopathy without any mention of the possibility of MND (45), suggesting that timely EDX testing does not always guarantee reduction in delay. 


\section{ADVANTAGES OF DECREASING TIME TO DIAGNOSIS IN ALS}

In addition to the numerous unnecessary, costly, and even painful procedures and investigations that arise from delayed or incorrect diagnosis, the results from this work up can be misinterpreted, leading to further prolongation of time to correct diagnosis. Overall, this unnecessary work up and often resultant surgical interventions may also result in delayed referrals to appropriate neuromuscular specialists and multidisciplinary clinics. In these clinics, patients are provided with the appropriate pharmacological and other supportive interventions, such as therapy (physical, occupational, speech and swallowing). Apart from diseasemodifying treatments, management involves symptomatic treatment that is specifically tailored to needs of ALS patients. Furthermore, these ALS centers allow for broader access to subspecialty evaluations and management in a single visit, thereby minimizing decentralized and often multiday appointments (46). Importantly, diagnosis and care of patients with ALS in such tertiary centers has been shown to decrease the frequency of hospital admissions, improve quality of life, and increase survival outcomes $(47,48)$. A more recent study in 2020 found that patients referred to ALS centers also experienced a significantly shorter diagnostic delay of 8.5 months compared to 12 months for those assessed at other facilities (16).

Delays to diagnosis may also affect enrollment into clinical trials and reducing the delay would allow for earlier initiation of disease-modifying treatment candidate agents and extended outcome monitoring periods. These clinical trials typically exclude patients further along in the disease course, as well as those with higher disease burden through the use of strict criteria such as conservative respiratory vital capacity cut-offs or limited timelines from symptom onset. One study assessing rates of exclusion in ALS clinic trials between 2000 and 2017 found that an average of $59.8 \%$ of patients are excluded. Respiratory function and disease duration were the second and third most common cited exclusion factors, respectively, with failure to meet a specific El Escorial category as the most common cause for exclusion (49).

Finally, earlier diagnosis and management of ALS can allow patients to more appropriately plan their futures regarding numerous aspects of their lives including financial, social, psychological, and spiritual. Patients will have more time to consider their own goals of medical care and make plans for their inevitable disability. Ultimately, an earlier diagnosis allows for more time to determine a meaningful and dignified end of life. Patients would have additional time to consider and document their own wishes with regard to artificial ventilation, feeding tube placement, and similar terminal care decisions. Furthermore, one cannot minimize the immense psychological toll that arises when a patient initially receives the incorrect diagnosis of a treatable or reversible disorder, only to be subsequently informed that their condition is actually a progressive and ultimately terminal disease. Such humanistic considerations should not be forgotten when formulating potential interventions to curb diagnostic delay. 


\section{POTENTIAL DISADVANTAGES OF DECREASING TIME TO DIAGNOSIS IN ALS}

Disadvantages to limiting diagnostic delay are largely theoretical but worth discussing, as the diagnosis of ALS remains mostly a clinical one and must be made after the exclusion of alternative and potentially treatable diseases. A 1999 article by Swash proposed a stepwise algorithm (Figure 2) for evaluating weakness and wasting as presenting clinical features, with the aim of minimizing diagnostic delay in ALS, while excluding mimicker conditions (50). He outlined two pathways, noting considerable overlap. The pathway comprising diagnosis by positive

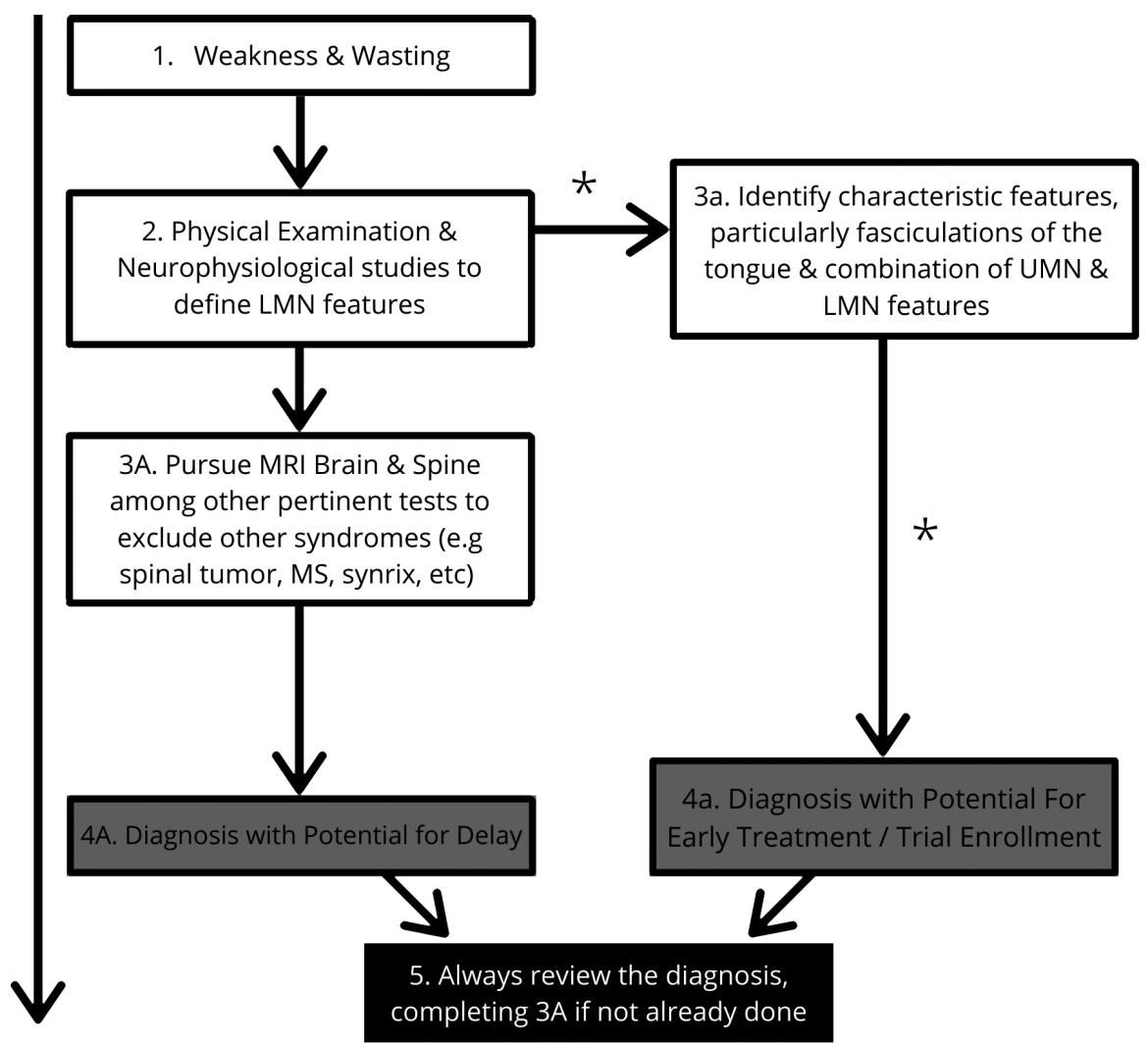

* = major rapid diagnostic pathway- a primarily clinical pathway which does not necessarily emphasize the specification of regions or extent of involvement MS= multiple sclerosis; UMN= upper motor neuron; LMN= lower motor neruon

Figure 2. A proposed neurological weakness and wasting workup algorithm, modified with permission from Swash (50). 
criteria is limited by the absence of a specific biologic diagnostic test. However, the other pathway comprising diagnosis by exclusion of other disorders may promote delay "that can be tempered only by efficiency in the investigative pathway".

One such potentially treatable condition that may present similarly as a progressive weakness is polyradiculopathy. If imaging is suggestive of such an etiology, surgery may be well-indicated. Therefore, shortening diagnostic delay may have the unintentional secondary effect of abating the extent of an appropriate work up for mimics and this may then result in failure to exclude other treatable conditions. Furthermore, it is possible for a patient to have both ALS and additional treatable and more commonly diagnosed neurological comorbidities such as peripheral neuropathy or carpal tunnel syndrome. Properly identifying and managing these conditions through thorough and appropriate investigations could result in improved quality of life, even if the patient is still ultimately diagnosed with ALS.

From a more humanistic perspective, a more extensive workup may ease some degree of psychological impact of receiving the news of a terminal diagnosis, particularly if the certainty of diagnosis is arrived at in a careful, stepwise fashion. This may better satisfy concerns on the part of both the physician and the patient, reassuring each party that no avenue of investigation has been left unaddressed.

\section{IMPLEMENTED MEASURES FOR MINIMIZING DIAGNOSTIC DELAY IN ALS}

In the United Kingdom (UK), a goal of the National Health Service (NHS) is to diagnose and initiate treatment of MND within 18 weeks of first referral from primary care providers. In January 2005, the Royal Preston Hospital in the UK introduced a 'fast-track' program for people suspected of having MND, with the ultimate goal to decrease wait times and allow for the final diagnosis to be given in an appropriate tertiary neurological/neuromuscular clinic-based environment. In a review of this program, the NHS goal was met in $91.9 \%$ of 'fast-track' patients compared to $57.1 \%$ of non-fast-track patients. Furthermore, the mean duration from referral to diagnosis was less than half as long for patients with the fast-track service compared to non-fast-track patients, 50 days compared to 104 days respectively. Interestingly, there was no definite improvement in mean time from initial symptom onset to diagnosis among ALS patients (collectively) after initiating the fast-track program, attributable to an insufficient number of patients through the fast-track pathway to impact the mean time to diagnosis in this specific ALS population (39).

\section{PROSPECTIVE OPPORTUNITIES TO MINIMIZE DIAGNOSTIC DELAY IN ALS}

Much of this chapter has discussed the role of primary care practice and its impact on length of time to the diagnosis of ALS. In an era of increasingly subspecialized 
medicine, PCPs are increasingly the "gatekeepers" of medicine (51). Unfortunately, a majority of PCPs will see, at most, only one or two ALS cases throughout their entire careers (52). Moreover, most general neurologists will only see a few cases of ALS per year (52) and their knowledge of classic ALS presentations may not be sufficient enough to make the correct diagnosis particularly in cases with more subtle disease onset, or complex presentations.

Intervention strategies similar to those used to improve dementia diagnoses (53), such as practice-based workshops and decision support software, could be applied to improve ALS detection rates at the gatekeeper level, and beyond. Similarly helpful may be diagnostic guidelines and algorithms embedded into electronic medical record software that could alert the user to ALS "red flag" symptoms, and prompt appropriate next steps. To this end, a recently published paper by Matharan et al. proposed an algorithm which may provide guidance regarding when to suspect ALS based on clinical signs and symptoms (Figure 3) (15).

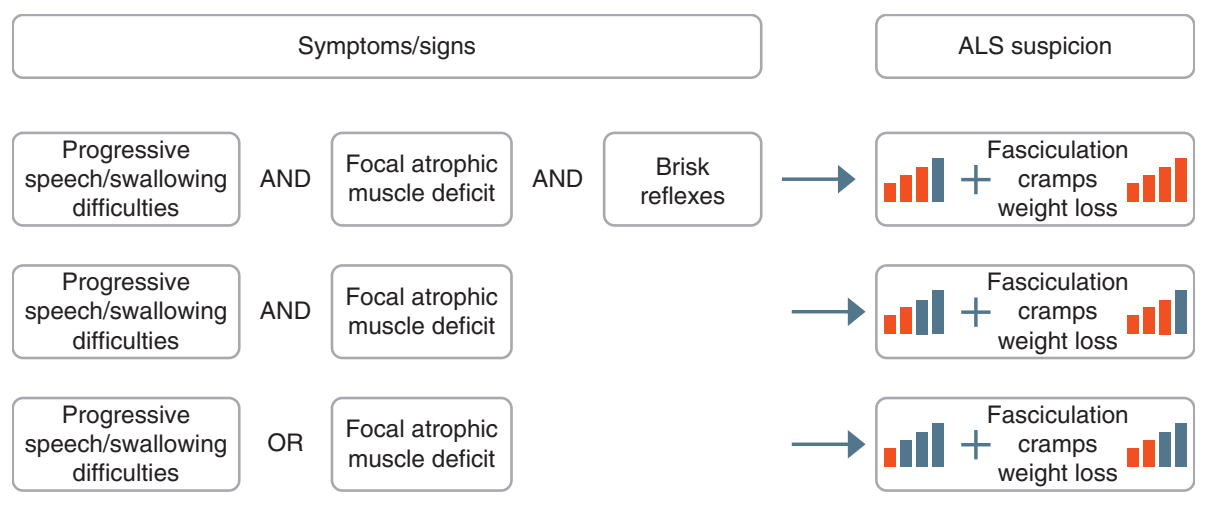

Figure 3. Proposed decision-making algorithm by Matharan et al. with the assistance of Graphandart.com to better screen for ALS based on clinical signs and symptoms (15).

However, ALS-specific provider education directed towards primary care physicians and general neurologists cannot be understated as a key method of intervention to minimize diagnostic errors. As noted in a 1999 paper by Eisen, a potential ALS surrogate marker is only effective if physicians are aware enough about the disease to consider ALS as a differential in the first place (52). Such education must focus on improving recognition of these "red flag" clinical features and correct interpretation of test results. One study found that $70 \%$ of patients who presented with a "red flag" symptom such as painless weakness, dysphagia, and gait disturbances did not have ALS as a differential diagnosis in their medical records (21). PCPs may seek a neurology consultation sooner, rather than manage a workup on their own. Furthermore, increased practitioner awareness regarding regional multidisciplinary centers would subsequently promote early referral as well. In addition, there should be education regarding available treatment options, both in terms of disease-modifying therapies and symptom-based management, as well as current clinical trials and those on the horizon, thereby limiting the potential component of "therapeutic nihilism" as noted in dementia literature (6). 
Education directed at the general public is also key. To raise the public awareness of ALS, one article proposed changing general terminology about the disease to more accessible phrasing for the average layperson, in much the same way strokes have in a sense been rebranded to "brain attack" and myocardial infarctions to "heart attacks". They also recommended increasing the number of ALS centers so as to be more accessible. However, they acknowledge that given the essential rarity of MNDs, such multidisciplinary centers are typically not costeffective by conventional standards. Therefore the article proposed creating broader-reaching neurodegenerative centers, where ALS patients may be treated alongside those with dementia, Parkinson's disease, or other progressive neurological conditions (52). Better public/patient understanding about common ALS symptoms may prompt the pursuit of medical evaluation sooner (public education in this regard will have to be necessarily tactful, so as to not promote too low of a threshold for concern). The public should be similarly educated on the availability of disease-modifying treatments and the necessity of intervening early in the disease process.

Ultimately, it is vital to determine where future improvements can be made along the ALS diagnostic timeline, including beyond the contribution of PCPs. Of course, many subsequent referrals are made to otorhinolaryngologists, orthopedists, rheumatologists, and neurosurgeons, among others, and future investigations and interventions pertinent to improving diagnostic delay would be remiss to not include these specialists as well.

\section{CONCLUSION}

Diagnostic delay impacts medicine across the spectrum of diseases and subspecialties, but even more so with a progressive neurodegenerative disease such as ALS. Current barriers to minimizing time to diagnosis include referrals to multiple specialists, misdiagnoses, and resultant unnecessary workups and procedures/ surgeries. These delays are particularly notable in patients with spine-onset ALS, for whom the differential diagnoses are typically broad.

There is marked potential to reduce these diagnostic delays through improved awareness and clinical education about ALS directed at primary care providers, as well as several other physician/provider types who evaluate these patients before definitive diagnosis is made. There is also a role for tailored education directed at the general public.

The recent literature review (10) found that the typical delay to diagnosis for ALS patients is 10-16 months reviewed studies from 1990 to 2020, which suggests the establishment of clinical diagnostic criteria and growing public awareness of ALS may not have been sufficient to significantly shorten delay (20). While this chapter has addressed the "what" and the "where" with regard to ALS diagnostic delay, there remains the question of why? Is there a reluctance by both PCPs and general neurologists to seek out second opinions from neuromuscular specialists and/or tertiary ALS multidisciplinary clinics? As mentioned previously, the dementia literature aptly notes a certain degree of "therapeutic nihilism" with regard to making the correct diagnosis. Could that also be a significant factor as it pertains to ALS? Perhaps physicians/providers experience some degree of 
apprehension in giving patients a terminal diagnosis when other avenues of additional investigation remain open, even if not fully warranted.

There remains an opportunity for broader awareness in the medical field about the role of neuromuscular specialists and tertiary centers in diagnosing and managing ALS. This may lessen the pursuit of unnecessary testing, procedures, and referrals, as may strategically educating the public on common signs and symptoms of the disease. Such education may ultimately result in more expedient referrals to ALS multidisciplinary clinics, followed by overall improvements to quality of life and longevity. Importantly, further dedicated research is needed at the patient and various provider levels regarding reducing the time to ALS diagnosis and hastening referrals to appropriate ALS specialists and multidisciplinary centers.

Conflict of interest: The authors declare no potential conflicts of interest with respect to research, authorship and/or publication of this manuscript.

Copyright and permission statement: The authors confirm that the materials included in this chapter do not violate copyright laws. Where relevant, appropriate permissions have been obtained from the original copyright holder(s), and all original sources have been appropriately acknowledged or referenced.

\section{REFERENCES}

1. Hardiman O, Van Den Berg LH, Kiernan MC. Clinical diagnosis and management of amyotrophic lateral sclerosis. Nat Rev Neurol. 2011;7(11):639-49. https://doi.org/10.1038/nrneurol.2011.153

2. Fuchs V, Kurppa K, Huhtala H, Collin P, Mäki M, Kaukinen K. Factors associated with long diagnostic delay in celiac disease. Scand J Gastroenterol. 2014;49(11):1304-10. https://doi.org/10.3109/00365 521.2014 .923502

3. Poon EG, Kachalia A, Puopolo AL, Gandhi TK, Studdert DM. Cognitive errors and logistical breakdowns contributing to missed and delayed diagnoses of breast and colorectal cancers: A process analysis of closed malpractice claims. J Gen Intern Med. 2012;27(11):1416-23. https://doi.org/10.1007/ sl1606-012-2107-4

4. Singh H, Sethi S, Raber M, Petersen LA. Errors in cancer diagnosis: Current understanding and future directions. J Clin Oncol. 2007;25(31):5009-18. https://doi.org/10.1200/JCO.2007.13.2142

5. Gandhi TK, Kachalia A, Thomas EJ, Puopolo AL, Yoon C, Brennan TA, et al. Missed and delayed diagnoses in the ambulatory setting: A study of closed malpractice claims. Ann Intern Med. 2006;145(7):488-96. https://doi.org/10.7326/0003-4819-145-7-200610030-00006

6. Aminzadeh F, Molnar FJ, Dalziel WB, Ayotte D. A review of barriers and enablers to diagnosis and management of persons with dementia in primary care. Can Geriatr J. 2012;15(3):85-94. https://doi. org/10.5770/cgj. 15.42

7. Koch T, Iliffe S. Rapid appraisal of barriers to the diagnosis and management of patients with dementia in primary care: A systematic review. BMC Fam Pract. 2010 Jul 1;11:52. https://doi. org/10.1186/1471-2296-11-52

8. Bradford A, Kunik ME, Schulz P, Williams SP, Singh H. Missed and delayed diagnosis of dementia in primary care: Prevalence and contributing factors. Alzheimer Dis Assoc Disord. 2009;23(4):306-14. https://doi.org/10.1097/WAD.0b013e318la6bebc

9. Woods B, Arosio F, Diaz A, Gove D, Holmerová I, Kinnaird L, et al. Timely diagnosis of dementia? Family carers' experiences in 5 European countries. Int J Geriatr Psychiatry. 2019;34(1):114-21 https://doi.org/10.1002/gps.4997 
10. Richards D, Morren JA, Pioro EP. Time to diagnosis and factors affecting diagnostic delay in amyotrophic lateral sclerosis. J Neurol Sci. 2020;417:117054. https://doi.org/10.1016/j.jns.2020.117054

11. Scialò C, Novi G, Bandettini di Poggio M, Canosa A, Sormani MP, Mandich P, et al. Clinical epidemiology of amyotrophic lateral sclerosis in Liguria, Italy: An update of LIGALS register. Amyotroph Lateral Scler Frontotemporal Degener. 2016;17(7-8):535-42. https://doi.org/10.1080/21678421.2016.1197 942

12. Yates E, Rafiq MK. Prognostic factors for survival in patients with amyotrophic lateral sclerosis: analysis of a multi-centre clinical trial. J Clin Neurosci. 2016;32:51-6. https://doi.org/10.1016/j. jocn.2015.12.037

13. Donaghy C, Dick A, Hardiman O, Patterson V. Timeliness of diagnosis in Motor Neurone Disease: A population-based study. Ulster Med J. 2008;77(1):18-21.

14. Armstrong MD, Hansen G, Schellenberg KL. Rural Residence and Diagnostic Delay for Amyotrophic Lateral Sclerosis in Saskatchewan. Can J Neurol Sci. 2020;47(4):538-542. https://doi.org/10.1017/ cjn. 2020.38

15. Matharan M, Mathis S, Bonabaud S, Carla L, Soulages A, Le Masson G. Minimizing the Diagnostic Delay in Amyotrophic Lateral Sclerosis: The Role of Nonneurologist Practitioners. Neurol Res Int. 2020; Article ID 1473981. https://doi.org/10.1155/2020/1473981

16. Martínez-Molina M, Argente-Escrig H, Polo MF, Hervás D, Frasquet M, Cortés V, et al. Early Referral to an ALS Center Reduces Several Months the Diagnostic Delay: A Multicenter-Based Study. Front Neurol. 2020;11:604922. https://doi.org/10.3389/fneur.2020.604922

17. Abdul Aziz NA, Toh TH, Goh KJ, Loh EC, Capelle DP, Abdul Latif L, et al. Natural history and clinical features of ALS in Malaysia. Amyotroph Lateral Scler Frontotemporal Degener. 2021;22(1-2):108-116. https://doi.org/10.1080/21678421.2020.1832121

18. Williams JR, Fitzhenry D, Grant L, Martyn D, Kerr DA. Diagnosis pathway for patients with amyotrophic lateral sclerosis: Retrospective analysis of the US Medicare longitudinal claims database. BMC Neurol. 2013;13:160. https://doi.org/10.1186/1471-2377-13-160

19. Hamidou B, Marin B, Lautrette G, Nicol M, Camu W, Corcia P, et al. Exploring the diagnosis delay and ALS functional impairment at diagnosis as relevant criteria for clinical trial enrolment. Amyotroph Lateral Scler Frontotemporal Degener. 2017;18(7-8):519-527. https://doi.org/10.1080/21678421.2 017.1353098

20. Cellura E, Spataro R, Taiello AC, La Bella V. Factors affecting the diagnostic delay in amyotrophic lateral sclerosis. Clin Neurol Neurosurg. 2012;114(6):550-4. https://doi.org/10.1016/j.clineuro.2011.11.026

21. Galvin M, Ryan P, Maguire S, Heverin M, Madden C, Vajda A, et al. The path to specialist multidisciplinary care in amyotrophic lateral sclerosis: A population-based study of consultations, interventions and costs. PLoS One. 2017;12(6):e0179796. https://doi.org/10.1371/journal.pone.0179796

22. Paganoni S, Macklin EA, Lee A, Murphy A, Chang J, Zipf A, et al. Diagnostic timelines and delays in diagnosing amyotrophic lateral sclerosis (ALS). Amyotroph Lateral Scler Frontotemporal Degener. 2014;15(5-6):453-6. https://doi.org/10.3109/21678421.2014.903974

23. Chio A. ISIS Survey: An international study on the diagnostic process and its implications in amyotrophic lateral sclerosis. J Neurol. 1999;246 Suppl 3:III1-5. https://doi.org/10.1007/BF03161081

24. Belsh JM, Schiffman PL. The amyotrophic lateral sclerosis (ALS) patient perspective on misdiagnosis and its repercussions. J Neurol Sci. 1996;139 Suppl:110-6. https://doi.org/ 10.1016/0022-510X(96)00088-3

25. Househam E, Swash M. Diagnostic delay in amyotrophic lateral sclerosis: What scope for improvement? J Neurol Sci. 2000;180(1-2):76-81. https://doi.org/10.1016/S0022-510X(00)00418-4

26. Zoccolella S, Beghi E, Palagano G, Fraddosio A, Samarelli V, Lamberti P, et al. Predictors of delay in the diagnosis and clinical trial entry of amyotrophic lateral sclerosis patients: A population-based study. J Neurol Sci. 2006;250(1-2):45-9. https://doi.org/10.1016/j.jns.2006.06.027

27. Kraemer M, Buerger M, Berlit P. Diagnostic problems and delay of diagnosis in amyotrophic lateral sclerosis. Clin Neurol Neurosurg. 2010;112(2):103-5. https://doi.org/10.1016/j.clineuro.2009.10.014

28. Kano O, Iwamoto K, Ito H, Kawase Y, Cridebring D, Ikeda K, et al. Limb-onset amyotrophic lateral sclerosis patients visiting orthopedist show a longer time-to-diagnosis since symptom onset. 2013; BMC Neurol 13, 19. https://doi.org/10.1186/1471-2377-13-19 
29. Rashed HR, Tork MA. Diagnostic delay among ALS patients: Egyptian study. Amyotroph Lateral Scler Frontotemporal Degener. 2020;21(5-6):416-19. https://doi.org/10.1080/21678421.2020.1763401

30. Turner MR, Scaber J, Goodfellow JA, Lord ME, Marsden R, Talbot K. The diagnostic pathway and prognosis in bulbar-onset amyotrophic lateral sclerosis. J Neurol Sci. 2010;294(1-2):81-5. https:// doi.org/10.1016/j.jns.2010.03.028

31. Vázquez-Costa JF, Martínez-Molina M, Fernández-Polo M, Fornés-Ferrer V, Frasquet-Carrera M, Sevilla-Mantecón T. Analysis of the diagnostic pathway and delay in patients with amyotrophic lateral sclerosis in the Valencian Community. Neurologia (Engl Ed). 2018;S0213-4853(18)30157-9.

32. Stevic Z, Kostic-Dedic S, Peric S, Dedic V, Basta I, Rakocevic-Stojanovic V, et al. Prognostic factors and survival of ALS patients from Belgrade, Serbia. Amyotroph Lateral Scler Frontotemporal Degener. 2016;17(7-8):508-14. https://doi.org/10.1080/21678421.2016.1195410

33. Knibb JA, Keren N, Kulka A, Leigh PN, Martin S, Shaw CE, et al. A clinical tool for predicting survival in ALS. J Neurol Neurosurg Psychiatry. 2016;87(12):1361-67. https://doi.org/10.1136/ jnnp-2015-312908

34. Calvo A, Moglia C, Lunetta C, Marinou K, Ticozzi N, Ferrante GD, et al. Factors predicting survival in ALS: a multicenter Italian study. J Neurol. 2017;264(1):54-63. https://doi.org/10.1007/ s00415-016-8313-y

35. Czaplinski A, Yen AA, Simpson EP, Appel SH. Slower disease progression and prolonged survival in contemporary patients with amyotrophic lateral sclerosis: Is the natural history of amyotrophic lateral sclerosis changing? Arch Neurol. 2006;63(8):1139-43. https://doi.org/10.1001/archneur.63.8.1139

36. Kacem I, Sghaier I, Bougatef S, Nasri A, Gargouri A, Ajroud-Driss S, et al. Epidemiological and clinical features of amyotrophic lateral sclerosis in a Tunisian cohort. Amyotroph Lateral Scler Frontotemporal Degener. 2020;21(1-2):131-139. https://doi.org/10.1080/21678421.2019.1704012

37. Nzwalo H, De Abreu D, Swash M, Pinto S, De Carvalho M. Delayed diagnosis in ALS: The problem continues. J Neurol Sci. 2014;343(1-2):173-5. https://doi.org/10.1016/j.jns.2014.06.003

38. Palese F, Sartori A, Logroscino G, Pisa FE. Predictors of diagnostic delay in amyotrophic lateral sclerosis: a cohort study based on administrative and electronic medical records data. Amyotroph Lateral Scler Frontotemporal Degener. 2019;20(3-4):176-185. https://doi.org/10.1080/21678421.2018.155 0517

39. Mitchell JD, Callagher P, Gardham J, Mitchell C, Dixon M, Addison-Jones R, et al. Timelines in the diagnostic evaluation of people with suspected amyotrophic lateral sclerosis (ALS)/motor neuron disease (MND)--a 20-year review: can we do better? Amyotroph Lateral Scler. 2010;11(6):537-41. https://doi.org/10.3109/17482968.2010.495158

40. Torny F, Lacoste M, Nguyen JM, Tymoczko-Nguyen ME, Couratier P. Étude des causes du retard au diagnostic de la sclérose latérale amyotrophique = Analysis of reasons for the late diagnosis of amylotrophic lateral sclerosis. Rev Neurol (Paris). 2006; https://doi.org/10.1016/S0035-3787(06)75055-X

41. Li TM, Swash M, Alberman E, Day SJ. Diagnosis of motor neuron disease by neurologists: A study in three countries. J Neurol Neurosurg Psychiatry. 1991;54(11):980-3. https://doi.org/10.1136/ jnnp.54.11.980

42. Belsh JM, Schiffman PL. Misdiagnosis in patients with amyotrophic lateral sclerosis. Arch Intern Med. 1990;150(11):2301-5. https://doi.org/10.1001/archinte.1990.00390220055011

43. Shayya L, Babu S, Pioro EP, Li J, Li Y. Distal Predominance of Electrodiagnostic Abnormalities in EarlyStage Amyotrophic Lateral Sclerosis. Muscle Nerve. 2018;58(3):389-395. https://doi.org/10.1002/ mus. 26158

44. Braun N, Macklin EA, Sinani E, Sherman A, Weber M. The revised El Escorial criteria "clinically probable laboratory supported ALS"-once a promising now a superfluous category? Amyotroph Lateral Scler Frontotemporal Degener. 2020;21(1-2):24-28. https://doi.org/10.1080/21678421.2019.1666 875

45. Srinivasan J, Scala S, Jones HR, Saleh F, Russell JA. Inappropriate surgeries resulting from misdiagnosis of early amyotrophic lateral sclerosis. Muscle Nerve. 2006;34(3):359-60. https://doi.org/10.1002/ mus. 20555 
46. Shook SJ, Pioro EP. Racing against the clock: Recognizing, differentiating, diagnosing, and referring the amyotrophic lateral sclerosis patient. Ann Neurol. 2009;65 Suppl 1:S10-6. https://doi.org/10.1002/ ana. 21545

47. Lee JRJ, Annegers JF, Appel SH. Prognosis of amyotrophic lateral sclerosis and the effect of referral selection. J Neurol Sci. 1995;132(2):207-15. https://doi.org/10.1016/0022-510X(95)00154-T

48. Chiò A, Bottacchi E, Buffa C, Mutani R, Mora G. Positive effects of tertiary centres for amyotrophic lateral sclerosis on outcome and use of hospital facilities. J Neurol Neurosurg Psychiatry. 2006;77(8):948-50. https://doi.org/10.1136/jnnp.2005.083402

49. Van Eijk RPA, Westeneng HJ, Nikolakopoulos S, Verhagen IE, Van Es MA, Eijkemans MJC, et al. Refining eligibility criteria for amyotrophic lateral sclerosis clinical trials. Neurology. 2019;92(5):e451-e460. https://doi.org/10.1212/WNL.0000000000006855

50. Swash M. An algorithm for ALS diagnosis and management. Neurology. 1999;53(8 Suppl 5):S58-62.

51. Gelinas D. Conceptual approach to diagnostic delay in ALS: A United States perspective. Neurology. 1999;53(8 Suppl 5):S17-21.

52. Eisen A. How to improve the diagnostic process. In: Journal of Neurology, Supplement. J Neurol. 1999;246 Suppl 3:III6-9. https://doi.org/10.1007/BF03161082

53. Koch $T$, Iliffe $S$. Dementia diagnosis andmanagement: A narrative review of changing practice. Br J Gen Pract. 2011;61(589):e513-25. https://doi.org/10.3399/bjgp11X588493 
